\title{
Métabolisme oxygéné des acides gras polyinsaturés et fonction dans les cellules sanguines et vasculaires ${ }^{1}$
}

\author{
Michel LAGARDE \\ Université de Lyon, UMR 1060 Inserm \\ (CarMeN, INFOLIP), IMBL, INSA \\ de Lyon, 20 avenue A. Einstein, \\ 69621 Villeurbanne \\ $<$ michel.lagarde@insa-lyon.fr>
}

\begin{abstract}
Most polyunsaturated fatty acids have the minimal 1,4-cis,cis-pentadiene configuration to be oxygenated into a conjugated diene hydroperoxide. This product is usually reduced into the corresponding hydroxy derivative by cell glutathione peroxidases. $A$ series of mono-, di- and tri-hydroxy derivatives can then be formed. In addition, eicosapolyenoic acids having at least three double bonds at carbons 8, 11 and 14 are oxygenated and cyclised by cyclooxygenases into prostanoids, as well as some docosapolyenoic counterparts with at least three double bonds at carbons 10,13 and 16, but to a lesser extent. Most of those oxygenated metabolites have been described with specific biological functions, especially in blood and vascular cells. Few cytochrome $P_{450}$ mono-oxygenases products have been described as well with less characterized biological functions.
\end{abstract}

Key words: oxygenases, prostanoids, leukotrienes, protectins, resolvins, octadecanoids, docosanoids

cette oxygénation. Cette libération a lieu principalement sous l'action de phospholipases $\mathrm{A}_{2}\left(\mathrm{PLA}_{2}\right)$, principalement $\mathrm{CPLA}_{2}$ qui est activée par phosphorylation de deux résidus Sérine et translocation de l'enzyme du cytosol à la membrane où l'hydrolyse de l'ester acyle en position sn2 (Balsinde et al., 2000). Elle concerne principalement les phosphatidyl-choline et -éthanolamine. Cette voie principale est parallèle à une voie quantitativement secondaire initiée par le clivage des phosphoinositides et de la phosphatidyl-choline par une phospholipase C suivie de l'hydrolyse séquentielle des diacylglycérols (DAG) résultants par les DAG lipase et monoacylglycérols (MAG) lipase. Cette voie indirecte conduit finalement à libérer aussi I'acide initialement en position sn-2 (Authi et al., 1985). Alors que la voie $\mathrm{CPLA}_{2}$ est assez spécifique du résidu arachidonoyle, la voie DAG/MAG lipases a la potentialité de libérer facilement d'autres AGPI (figure 1). appelée auto-oxydation ou peroxydation, par exemple associée au diabète et au vieillissement.

Pour cette oxygénation dépendante des di-oxygénases, les AGPI doivent d'abord être libérés des glycéro-phospholipides membranaires des cellules siège de

\footnotetext{
${ }^{1}$ Conférence Chevreul prononcée à Munich, dans le cadre du Congrès Euro Fed Lipid le 22 novembre 2010.
}

\section{Oxygénation de I'acide arachidonique (ARA) comme référence : formation d'eicosanoïdes}

L'oxygénation spécifique de l'ARA par les cyclo-oxygénases (Cox) et lipo-oxygénases (Lox) est bien décrite en ayant fait l'objet de très nombreuses études et revues (Lagarde, 1988 ; Smith, 2008). Cette oxygénation dépend essentiellement de l'équipement enzymatique des cellules concernées par l'activité des produits oxygénés (prostanoïdes, leucotriènes et produits apparentés, collectivement nommés eicosanoïdes).

La Cox constitutive (Cox-1) est assez ubiquiste alors que l'expression de la Cox inductible (Cox-2) est plus restreinte et concerne notamment les cellules impliquées dans les phénomènes inflammatoires. Les deux formes conduisent à la formation de prostaglandine (PG) $\mathrm{G}_{2}$, facilement transformée en $\mathrm{PGH}_{2}$ par l'activité peroxydase associée à la protéine Cox, d'où l'autre nom de PGH synthase donné à l'enzyme. L'isomérisation de la $\mathrm{PGH}_{2}$ en prostaglandines primaires $\mathrm{PGD}_{2}, \mathrm{PGE}_{2}$ et $\mathrm{PGF}_{2 \alpha}$ est également assez ubiquiste. En partie spontanée pour $\mathrm{PGD}_{2}$ et $\mathrm{PGE}_{2}$, elle est aussi catalysée par les PGE et PGD synthases, alors que la formation de $\mathrm{PGF}_{2 \alpha}$ requiert une $\mathrm{PGH}$ réductase. À l'inverse, l'isomérisation de la $\mathrm{PGH}_{2}$ en thromboxane $\mathrm{A}_{2}$ ou en $\mathrm{PGI}_{2} /$ prostacycline est beaucoup plus spécifique: dans les plaquettes sanguines (Patrono, 1989) pour le premier et la paroi vasculaire (endothélium et cellules musculaires lisses) (Smith, 1986) ainsi que le lymphocyte (Wu et al., 1987) pour la deuxième. Cette isomérisation est de type cytochrome $P_{450}$ dans sa fonction isomérase et 


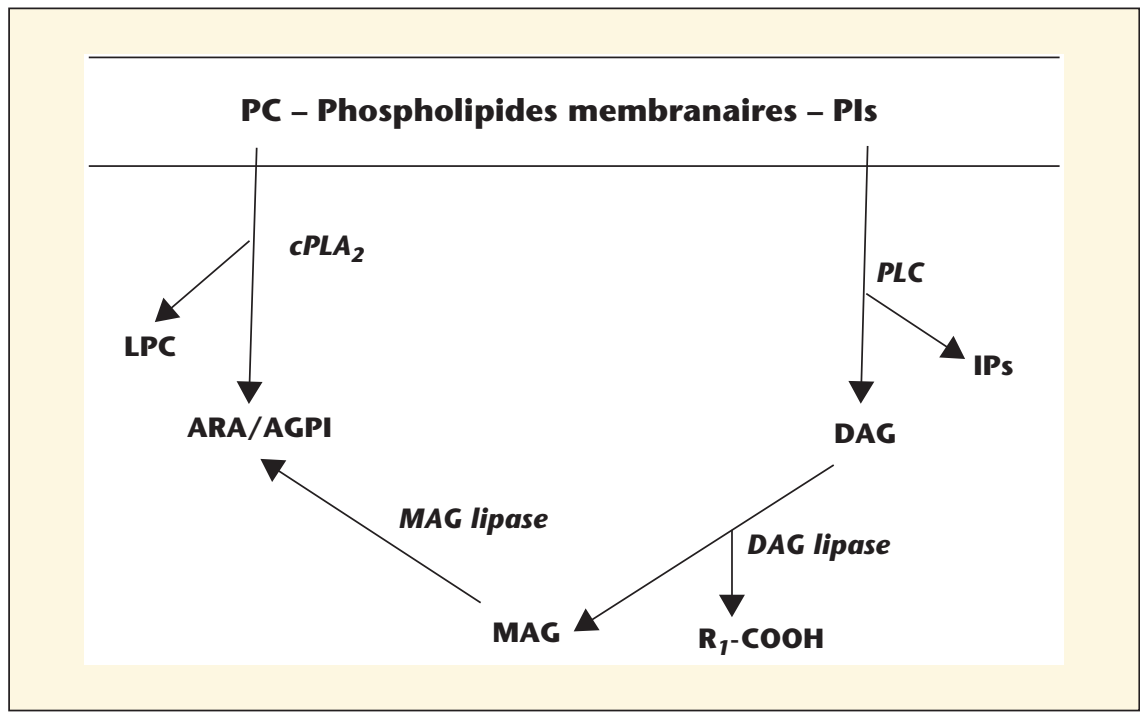

Figure 1. Voies de libération de l'acide arachidonique (ARA) ou d'autres acides gras polyinsaturés (AGPI) à partir des phospholipides membranaires, principalement phosphatidylcholine (PC) et phosphoinositides (PIs), respectivement par la phospholipase $A_{2}$ cytosolique $\left(C P L A_{2}\right)$ et la cascade phospholipase C (PLC)/diacyl/monoacyl glycérol (DAG/MAG) lipase. La CPLA2 est transférée du cytosol à la membrane après phosphorylation.

caractérisée comme telle pour la thromboxane synthase $(T x-S)$ et la prostacycline synthase (PGI-S) (Hecker et Ullrich, 1989). Les effets biologiques marquants de $\mathrm{TxA}_{2}$ sont I'induction de l'agrégation plaquettaire et la vasoconstriction alors que ceux de la $\mathrm{PGI}_{2}$ sont exactement

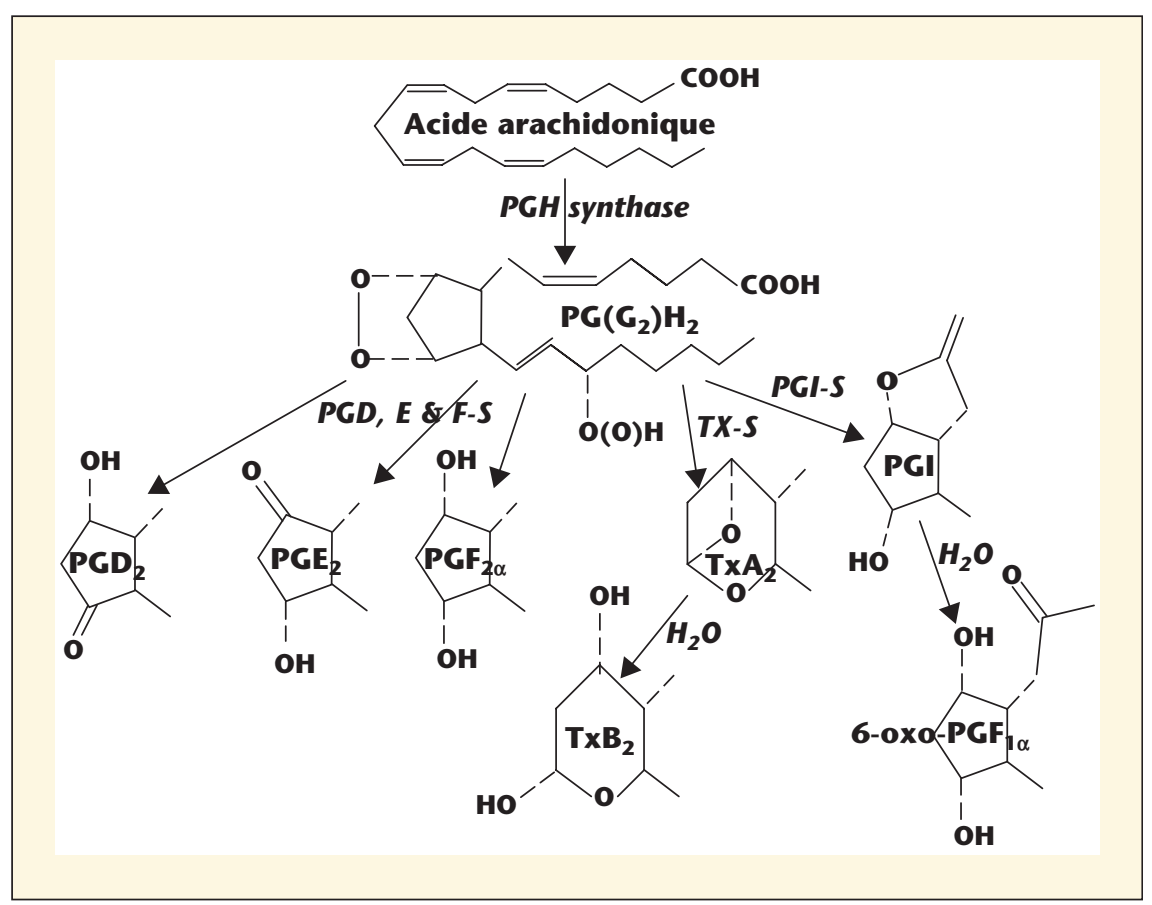

Figure 2. Résumé des voies de biosynthèse des prostanoïdes de la série 2 à partir d'ARA via les prostaglandines (PG) $H$ synthases, encore appelées cyclooxygénases (Cox-1 et -2), et les isomérases/réductases que sont les $P G D, E, F, I$ synthases et la thromboxane (Tx) synthase. été la 12-Lox, décrite dans les plaquettes, conduisant au 12-hydroperoxy-eicosatétraénoate (12-HpETE) (Hamberg et Samuelsson, 1974). Cet hydroperoxyde est normalement réduit par une glutathion peroxydase en équivalent 12hydroxyde appelé 12-HETE (Bryant et Bailey, 1981). La 15-Lox conduit de même au 15-HpETE puis au 15-HETE à partir d'ARA (Vanderhoek, 1988). Cette conversion est identique à celle décrite auparavant dans le règne végétal pour l'oxygénation de l'acide linoléique en 13 hydroperoxy-octadécadiénoïque ou 13HpODE (Hamberg, 1971). La troisième Lox animale a fait l'objet de beaucoup plus de développements en raison des retombées physiopathologiques de ses produits : les leucotriènes; il s'agit de la 5-Lox conduisant au 5-HpETE, qu'elle convertit aussi en dérivé époxyde, le leucotriène (LT) $\mathrm{A}_{4}$ (Borgeat, 1989). La 5Lox est donc une enzyme bi-fonctionnelle qui requiert en outre du calcium pour son fonctionnement (Werz et al., 2002), ce qui est beaucoup moins évident pour les 12- et 15-Lox. Le LTA est une molécule sans activité biologique connue mais destinée à hydrolyse en $\mathrm{LTB}_{4}$ et condensation avec une molécule de glutathion réduit pour conduire au $\mathrm{LTC}_{4}$, premier leucotriène peptidique, ultérieurement transformé en $\mathrm{LTD}_{4}$ puis $\mathrm{E}_{4}$ (Kuo et al., 1984) (figure 3).

Plus récemment, la mono-oxygénation de I'ARA par des cytochromes $P_{450}$, produisant des dérivés époxydes (époxy-eicosatriénoates ou EET) sur chaque double liaison à ses dépens, a été décrite. Ainsi, quatre époxygénases produisent les 5,6-EET, 8,9-EET, 11,12EET ou 14,15-EET (Oliw, 1994). Des époxydes hydrolases conduisent aux dérivés di-hydroxylés vicinaux respectifs, exemple : 5,6-diHETE. Des monooxygénases à cytochrome $\mathrm{P}_{450}$ hydroxylant I'ARA en positions oméga et oméga-1 ont également été décrites (Makita et al., 1996). La figure 4 schématise ces voies biosynthétiques. Les activités biologiques de tous ces composés ne sont encore que partiellement évaluées; ils apparaissent dotés d'effets vasoactifs (Capdevila et al., 2000, 2007).

\section{Oxygénation des autres précurseurs de prostanoïdes}

Les autres précurseurs de prostanoïdes que sont l'acide dihomogamma-linol- 


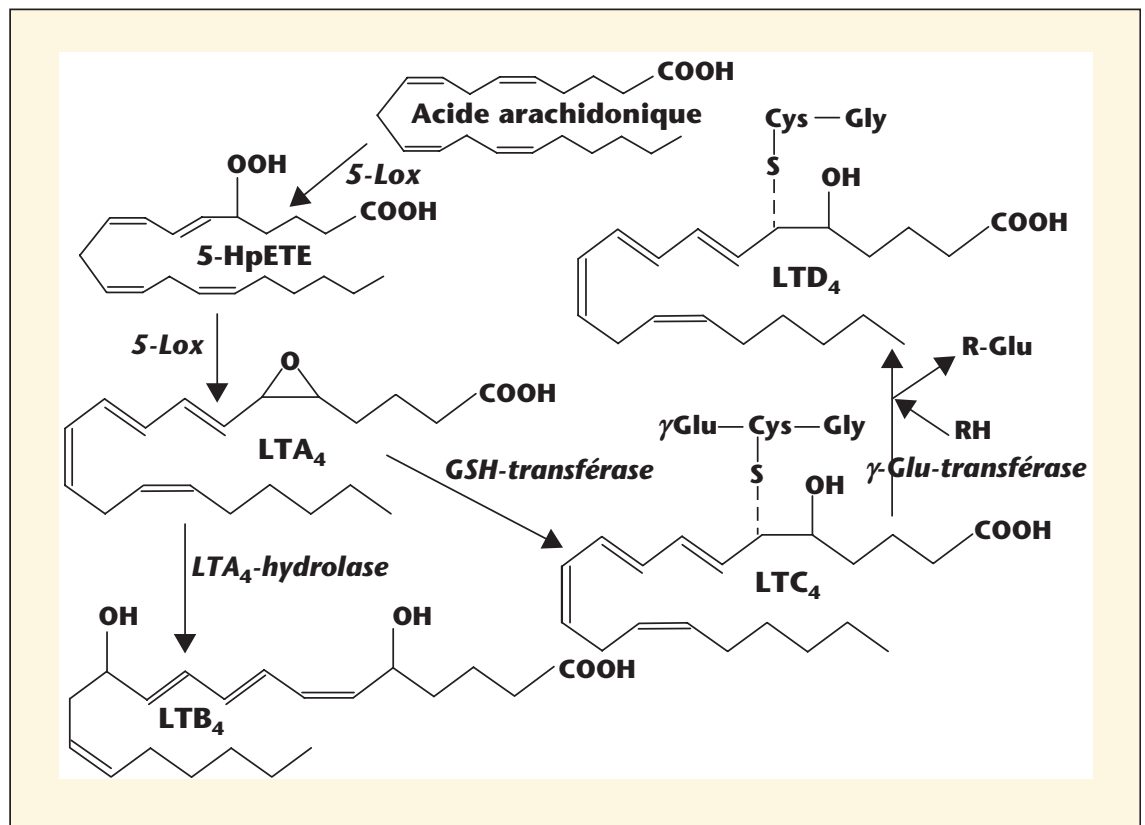

Figure 3. Voies de synthèse des leucotriènes (LT) à partir d'ARA, jusqu'au LTD 4. 5-HpETE : 5hydroperoxy-eicosatétraénoate; GSH: gluthation réduit; Cys, Gly et Glu: respectivement résidus cystéine, glycine et glutamate.

énique (DGLA ou 20:3ळ6) et I'acide eicosapentaénoïque (EPA ou 20:5ळ3) conduisent en théorie aux prostanoïdes, respectivement des séries 1 et 3 (exemples : $P \mathrm{PE}_{1}$ et $P \mathrm{PE}_{3}$ ). Une étude comparative de la formation des prostanoïdes des séries 1, 2 et 3 a montré que la Tx-S transforme très mal $\mathrm{PGH}_{1}$ et $\mathrm{PGH}_{3}$ dans les plaquettes, sauf en présence d'ARA ou de son produit de 12-Lox, 12-HpETE, ce qui révèle le requis en peroxydes de la voie Cox/Tx-S (Boukhchache et Lagarde, 1982). Ce requis est particulièrement flagrant pour l'oxygénation de I'EPA, aussi bien par la voie Cox/Tx-S que la voie 12-Lox (Boukhchache et Lagarde, 1982 ; Morita et al., 1983). La formation de $\mathrm{PGI}_{3}$ requiert également des peroxydes qui

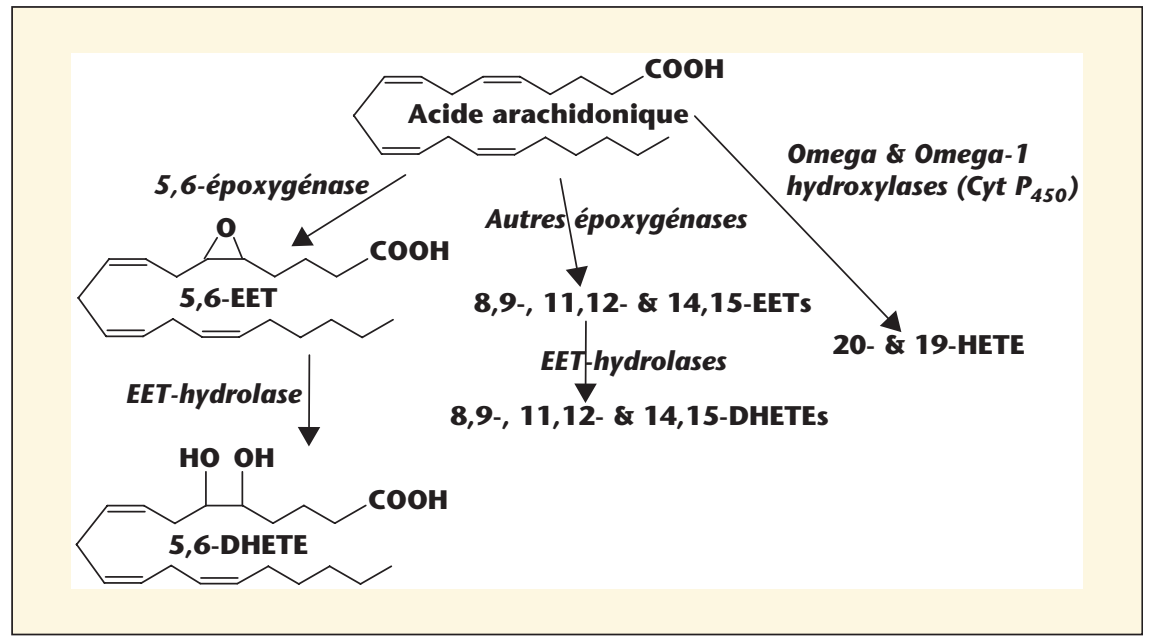

Figure 4. Résumé des voies de formation de dérivés hydroxylés de I'ARA via la monooxygénation par des enzymes à cytochrome $P_{450}$. DHETE : dihydroxytriénoate, EET : époxy-eicosatriénoate; HETE : hydroxyeicosatétraénoate. peuvent être apportés par le produit de Lox comme le 15-HpETE, pertinent au niveau de I'endothélium vasculaire qui possède la 15-Lox (Bordet et al., 1986). Ceci indique le même requis en peroxydes pour la voie Cox/PGI-S.

Le produit d'élongation de I'ARA, I'acide docosatétraénoïque (22:4ळ6) ou acide adrénique, peut aussi être oxygéné par la voie Cox/Tx-S/PGI-S en dihomo-thromboxane et -prostacycline (VanRollins et al., 1985) dont les activités biologiques sont respectivement celles de $\mathrm{TxA}_{2}$ et $\mathrm{PGI}_{2}$ mais à un moindre degré. Comme la formation de $\mathrm{PGI}_{3}$, celle de dihomo- $\mathrm{PGI}_{2}$ est très dépendante de peroxydes, dont ceux issus de la 15-Lox (Bordet et al., 1988).

Un isomère de position du DGLA/20:3ळ6 ayant une pertinence biologique à la fois comme marqueur des déficits en acides gras $\varpi 6$ et dans les régimes riches en acides gras saturés (Renaud, 1974), I'acide de Mead ou 20:3ळ9, n'est pas précurseur de prostanoïdes car il ne possède pas les trois double liaisons minimales sur les carbones 8,11,14, il est cependant substrat de la 12-Lox plaquettaire (Jakschik et al., 1983 ; Lagarde et al., 1983). La formation de 12-OH-5,8,10-20:3 est associée à un contrôle bimodal de l'agrégation plaquettaire qui mime celui induit par la $\mathrm{PGE}_{2}$ (Lagarde et al., 1985). Cette formation est également stimulée par le $12 \mathrm{HpETE}$, ce qui confirme le requis de la 12-Lox, comme la Cox, en peroxydes physiologiques (Croset et Lagarde, 1985). La figure 5 résume l'oxygénation par les Cox et Lox d'analogues et homogues de I'ARA.

\section{Formation d'octadécanoïdes}

Les octadécanoïdes pertinents sont ceux issus des acides linoléique (LA ou 18:2ळ6) et linolénique (LNA ou 18:3ळ3) en raison de la valeur nutritionnelle de ces précurseurs.

Le dérivé hydroxylé de LA issu de la voie 15/ळ6-Lox après réduction par la glutathion peroxydase : le 13-OH-18:2, appelé 13-HODE (13-Hydroxy-octadécadiénoate), a été caractérisé dans plusieurs systèmes biologiques, notamment I'endothélium vasculaire où son rôle inhibiteur de l'adhésion plaquettaire a été souligné (Buchanan et al., 1985). L'autre dérivé possible est le 9-HODE qui a été décrit comme un produit 


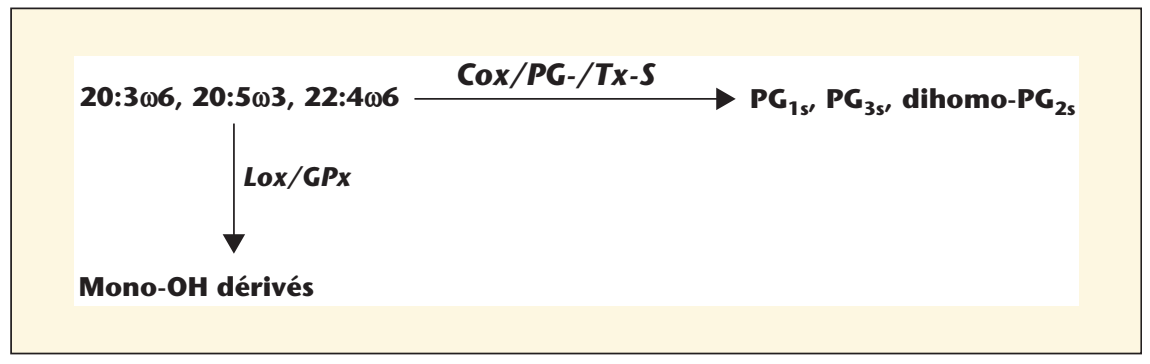

Figure 5. Résumé de la dioxygénation des principaux analogues et homologues de I'ARA par la voie Cox et lipoxygénase (Lox). GPx : glutathion peroxydase associées aux Lox.

d'oxygénation partielle de LA par la Cox (Kaduce et al., 1989). Le 9-HODE a aussi été décrit comme un marqueur d'oxydation in vivo au même titre que le 13HODE (Colas et al., 2010).

Le métabolisme oxygéné le plus connu de LNA est celui conduisant à l'acide jasmonique, un dérivé cyclique dont la formation est initiée par la 15-/ळ6-Lox chez les végétaux. Ce dérivé possède des activités bien identifiées de résistance aux phyto-pathogènes (Blée, 2002). Un produit de cyclo-oxygénation incomplète du LNA par la Cox-2 a également été décrit comme le 12-OH-9,11,1518:3 (Laneuville et al., 1995), mais aucune activité biologique $n^{\prime} y$ a été associée. La figure 6 montre la formation des principaux octadécanoïdes.

\section{Oxygénation de l'acide docosahexaénoïque}

L'acide docosahexaénoïque (DHA ou 22:6 63 ) est un des acides gras les plus abondants entrant dans la composition des lipides marins. C'est également I'AGPI majeur du cerveau et de la rétine, retrouvé aussi en quantités importantes dans les spermatozoïdes (Salem et al., 2001). Il est par ailleurs un des éléments importants de la protection cardiovasculaire observée suite à l'ingestion de lipides marins, conjointement à I'EPA (Leaf, 1992).

Les premiers travaux portant sur les métabolites oxygénés du DHA ont rapidement montré que cet $A G P I$ n'est pas transformé en dihomo-prostanoïdes

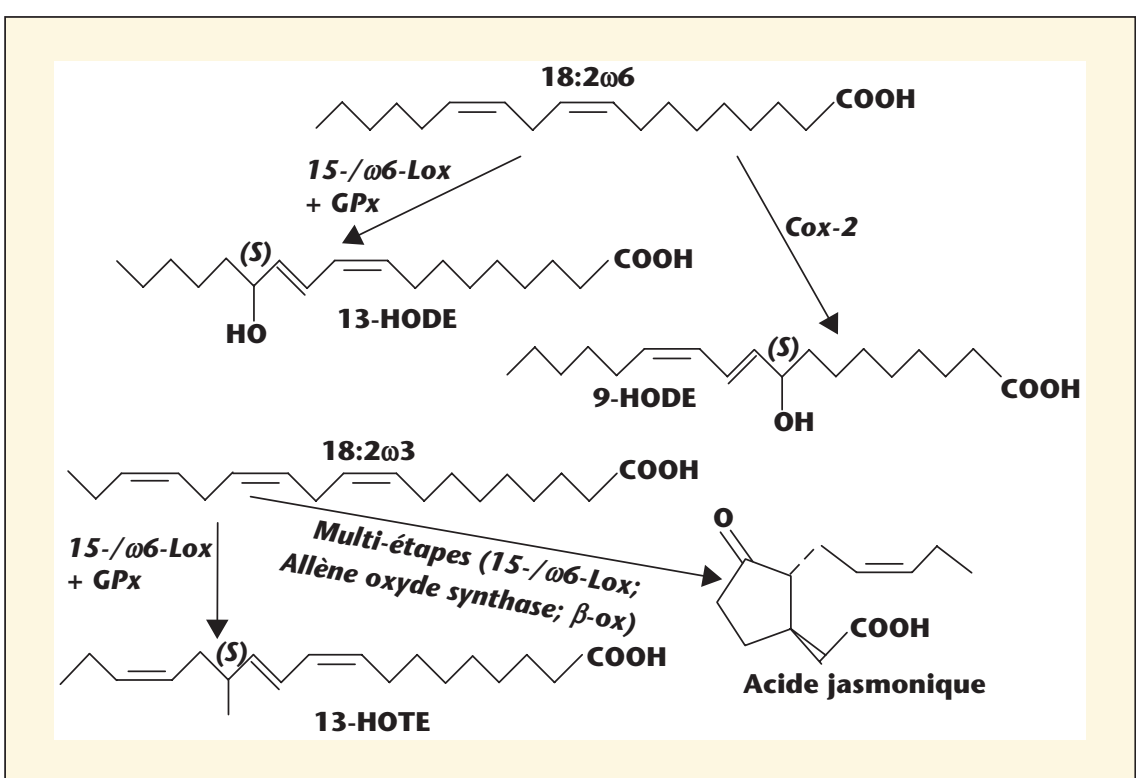

Figure 6. Schéma d'oxygénation principale des deux acides gras essentiels linoléique (18:2ळ6) et linolénique (18:3ळ3) via la 15-/ळ6-Lox. HODE : hydroxyoctadécadiénoate; HOTE : hydroxyoctadécatriénoate. par les Cox mais oxygéné par diverses lipoxygénases (Corey et al., 1983). La multiplicité des doubles liaisons conduit à une plus grande variété de dérivés hydroxylés par réduction des hydroperoxydes formés que I'ARA. À titre d'exemple la 12-/ळ9-Lox conduit aux 11-OH et 14-OH-22:6 (Aveldaño et Sprecher, 1983) et la 5-Lox aux 4-OH et 7-OH-22:6 (Lee et al., 1984 ; Yamamoto et al., 2008). Des travaux récents montrent que le DHA subit même une double lipoxygénation par la 15-/ळ6-Lox (Chen et al., 2009) et que le produit dihydroxylé correspondant, appelé PDX, inhibe I'agrégation plaquettaire via une inhibition de la Cox-1 et de la réponse au $\mathrm{TxA}_{2}$. Cette propriété est due à la géométrie particulière du triène conjugué formé par double lipoxygénation, géométrie trans,cis,trans partagée par d'autres produits de double lipoxygénation d'AGPI collectivement appelés " poxytrins" (Chen et al., 2011). Les dérivés mono-hydroxylés du DHA inhibent l'agrégation des plaquettes sanguines induite par le $\mathrm{TxA}_{2}$ plus puissamment que ne le font les dérivés correspondants d'ARA (Croset et al., 1988).

Une série de produits di- et tri-hydroxylés du DHA ont été décrits comme de puissants inhibiteurs de I'inflammation, en la prévenant ou accélérant sa résolution. Ces molécules sont appelées marésines (Serhan et al., 2009), résolvines D et protectines (Ariel et Serhan, 2007) ; leur voies biosynthétiques simplifiées sont représentées dans la figure 7. La protectine D1 est aussi appelée neuroprotectine D1 en raison de ses propriétés inhibitrices de l'apoptose neuronale (Lukiw et Bazan, 2010). Des dérivés analogues avec des propriétés similaires sont produits à partir d'EPA; ils ont été appelés résolvines $E$. De même, le marqueur de déficit en acides gras oméga-3, l'acide docosapentaénoïque ๘6, DPAळ6, conduit à des dérivés hydroxylés anti-inflammatoires (Dangi et al., 2009), en plus de produits de Cox (Milks et Sprecher, 1985) non évalués en termes d'activité biologique.

\section{Conclusion}

Au-delà de l'acide arachidonique, vu comme un AGPI de référence, la plupart des autres AGPI peuvent être oxygénés par les monooxygénases à cytochrome $\mathrm{P}_{450}$ et les dioxygénases ( $\mathrm{PGH}$ synthases et lipoxygénases) en dérivés bioactifs, 


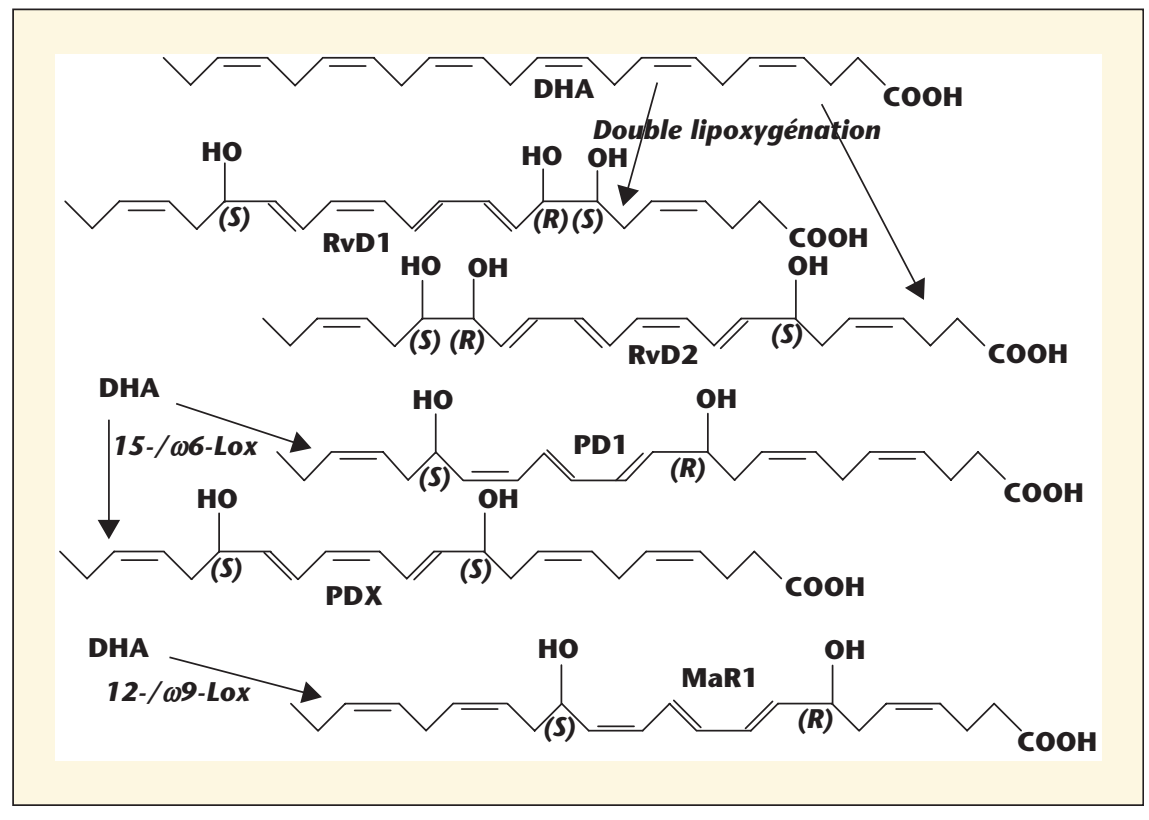

Figure 7. Schéma de formation des principaux docosanoïdes d'intérêt biologique à partir d'acide docosahexaénoïque (DHA) via plusieurs lipoxygénases. Mar : marésine; PD : protectine $D ; R v D$ : résolvine $D$.

notamment dans le secteur vasculaire. Leur formation est largement soumise à compétition ou potentialisation entre les différents AGPI précurseurs. Les différents dérivés oxygénés produits, appelés eicosanoïdes, octadécanoïdes et docosanoïdes, ont des actions très spécifiques qui dépendent largement des cellules cibles et de leur équipement en récepteurs aux dérivés. Le champ de recherche récemment ouvert sur les docosanoïdes, notamment ceux dérivés du DHA, est en plein développement dans le secteur cérébrovasculaire.

Remerciements. L'auteur remercie I'Inserm et le ministère de l'Enseignement supérieur et de la recherche pour leur support, ainsi que les Collègues impliqués dans les travaux de son équipe, notamment le Professeur Michel Guichardant.

\section{RÉFÉRENCES}

Ariel A, Serhan CN. Resolvins and protectins in the termination program of acute inflammation. Trends Immunol 2007 ; 28 : 176-83.

Aveldaño MI, Sprecher $H$. Synthesis of hydroxy fatty acids from $4,7,10,13,16$, 19-[1-14C] docosahexaenoic acid by human platelets. J Biol Chem 1983 ; 258 : 9339-43.
Authi KS, Lagarde M, Crawford N. Diacylglycerol lipase activity in human platelet intracellular and surface membranes. Some kinetic properties and fatty acid specificity. FEBS Lett $1985 ; 180$ : 95-101.

Balsinde J, Balboa MA, Dennis EA. Group IV cytosolic phospholipase A2 activation by diacylglycerol pyrophosphate in murine P388D1 macrophages. Ann N Y Acad Sci $2000 ; 905: 11-5$.

Blée E. Impact of phyto-oxylipins in plant defense. Trends Plant Sci $2002 ; 7$ : 315-22.

Bordet JC, Guichardant M, Lagarde M. Arachidonic acid strongly stimulates prostaglandin I3 (PGI3) production from eicosapentaenoic acid in human endothelial cells. Biochem Biophys Res Commun 1986; 135 : 403-10.

Bordet JC, Guichardant M, Lagarde M. Hydroperoxides produced by $n-6$ lipoxygenation of arachidonic and linoleic acids potentiate synthesis of prostacyclin related compounds. Biochim Biophys Acta 1988 ; 958: 460-8.

Borgeat P. Biochemistry of the lipoxygenase pathways in neutrophils. Can J Physiol Pharmacol 1989 ; 67 : 936-42.

Boukhchache D, Lagarde M. Interactions between prostaglandin precursors during their oxygenation by human platelets. Biochim Biophys Acta 1982 ; 713 : 386-92.

Bryant RW, Bailey JM. Role of seleniumdependent glutathione peroxidase in platelet

lipoxygenase metabolism. Prog Lipid Res $1981 ; 20$ : 189-94.

Buchanan MR, Haas TA, Lagarde $M$, Guichardant M. 13-Hydroxyoctadecadienoic acid is the vessel wall chemorepellant factor LOX. J Biol Chem 1985 ; 260 : 16056-9.

Capdevila JH, Falck JR, Harris RC. Cytochrome P450 and arachidonic acid bioactivation Molecular and functional properties of the arachidonate monooxygenase. I Lipid Res $2000 ; 41: 163-81$.

Capdevila JH, Falck JR, Imig JD. Roles of the cytochrome P450 arachidonic acid monooxygenases in the control of systemic blood pressure and experimental hypertension. Kidney Int 2007 ; 72 : 683-9.

Chen $P$, Véricel $E$, Lagarde $M$, Guichardant M. Poxytrins, a class of oxygenated products from polyunsaturated fatty acids, potently inhibit blood platelet aggregation. FASEB $2011 ; 25$ : 382-8.

Corey EJ, Shih C, Cashman JR. Docosahexaenoic acid is a strong inhibitor of prostaglandin but not leukotriene biosynthesis. Proc Natl Acad Sci U S A $1983 ; 80$ : 3581-4.

Croset M, Lagarde M. Enhancement of eicosaenoic acid lipoxygenation in human platelets by 12-hydroperoxy derivative of arachidonic acid. Lipids $1985 ; 20$ : 743-50.

Colas R, Pruneta-Deloche V, Guichardant M, et al. Increased lipid peroxidation in LDL from type- 2 diabetic patients. Lipids 2010 ; 45 : 723-31.

Croset M, Sala A, Folco G, Lagarde M. Inhibition by lipoxygenase products of TXA2-like responses of platelets and vascular smooth muscle 14 -Hydroxy from $22: 6 n-3$ is more potent than 12-HETE. Biochem Pharmacol $1988 ; 37: 1275-80$.

Dangi $B$, Obeng $M$, Nauroth JM, et al. Biogenic synthesis, purification, and chemical characterization of anti-inflammatory resolvins derived from docosapentaenoic acid (DPAn-6). J Biol Chem 2009 ; 284 : 14744-59.

Hamberg M. Steric analysis of hydroperoxides formed by lipoxygenase oxygenation of linoleic acid. Anal Biochem 1971 ; 43 : 515-26.

Hamberg M, Samuelsson B. Prostaglandin endoperoxides Novel transformations of arachidonic acid in human platelets. Proc Natl Acad Sci U S A 1974 ; 71 : 3400-4.

Hecker M, Ullrich V. On the mechanism of prostacyclin and thromboxane $\mathrm{A} 2$ biosynthesis. J Biol Chem $1989 ; 264$ : 141-50.

Jakschik BA, Morrison AR, Sprecher H. Products derived from 5,8,11-eicosatrienoic acid by the 5-lipoxygenase-leukotriene pathway. J Biol Chem 1983 ; 258 : 12797-800.

Kaduce TL, Figard PH, Leifur R, Spector AA. Formation of 9-hydroxyoctadecadienoic acid 
from linoleic acid in endothelial cells. J Biol Chem $1989 ; 264$ : 6823-30.

Kuo CG, Lewis MT, Jakschik BA. Leukotriene D4 and E4 formation by plasma membrane bound enzymes. Prostaglandins $1984 ; 28$ : 929-38.

Lagarde M. Metabolism of fatty acids by platelets and the functions of various metabolites in mediating platelet function. Prog Lipid Res $1988 ; 27$ : 135-52.

Lagarde M, Burtin M, Rigaud M, Sprecher H, Dechavanne M, Renaud S. Prostaglandin E2like activity of 20:3n-9 platelet lipoxygenase end-product. FEBS Lett $1985 ; 181$ : 53-6.

Lagarde $M$, Burtin $M$, Sprecher $H$, Dechavanne $M$, Renaud $S$. Potentiating effect of 5,8,11-eicosatrienoic acid on human platelet aggregation. Lipids $1983 ; 18$ : 291-4.

Laneuville O, Breuer DK, Xu N, et al. Fatty acid substrate specificities of human prostaglandin-endoperoxide $\mathrm{H}$ synthase- 1 and -2 Formation of 12-hydroxy-(9Z, 13E/Z, 15Z)octadecatrienoic acids from alpha-linolenic acid. J Biol Chem 1995 ; 270 : 19330-6.

Leaf A. Health claims: omega-3 fatty acids and cardiovascular disease. Nutr Rev 1992 ; $50: 150-4$.

Lee TH, Mencia-Huerta JM, Shih C, Corey EJ, Lewis RA, Austen KF. Effects of exogenous arachidonic, eicosapentaenoic, and docosahexaenoic acids on the generation of 5-lipoxygenase pathway products by ionophore-activated human neutrophils. J Clin Invest $1984 ; 74: 1922-33$.
Lukiw W], Bazan NG. Inflammatory, apoptotic, and survival gene signaling in Alzheimer's disease A review on the bioactivity of neuroprotectin D1 and apoptosis. Mol Neurobiol $2010 ; 42$ : 10-6.

Makita K, Falck JR, Capdevila JH. Cytochrome P450, the arachidonic acid cascade, and hypertension: new vistas for an old enzyme system. FASEB / $1996 ; 10$ : 1456-63.

Milks MM, Sprecher H. Metabolism of 4,7, 10,13,16-docosapentaenoic acid by human platelet cyclooxygenase and lipoxygenase. Biochim Biophys Acta 1985 ; 835 : 29-35.

Morita I, Takahashi R, Saito Y, Murota S. Stimulation of eicosapentaenoic acid metabolism in washed human platelets by 12 hydroperoxyeicosatetraenoic acid. J Biol Chem 1983 ; 258 : 10197-9.

Oliw EH. Oxygenation of polyunsaturated fatty acids by cytochrome P450 monooxygenases. Prog Lipid Res 1994 ; 33 : 329-54.

Patrono C. Aspirin and human platelets: from clinical trials to acetylation of cyclooxygenase and back.n Trends Pharmacol Sci 1989; 10 : 453-8.

Renaud S. Dietary fats and atherosclerosis in rat and rabbit. Adv Cardiol 1974 ; 13 : 169-82.

Salem Jr N, Litman B, Kim HY, Gawrisch K. Mechanisms of action of docosahexaenoic acid in the nervous system. Lipids $2001 ; 36$ : 945-59.

Serhan CN, Yang R, Martinod K, et al. Maresins: novel macrophage mediators with potent antiinflammatory and proresolving actions. J Exp Med 2009 ; 206 : 15-23.

Smith WL. Prostaglandin biosynthesis and its compartmentation in vascular smooth muscle and endothelial cells. Annu Rev Physiol $1986 ; 48: 251-62$.

Smith WL. Nutritionally essential fatty acids and biologically indispensable cyclooxygenases. Trends Biochem Sci 2008; 33 : 27-37.

Vanderhoek JY. Role of the 15-lipoxygenase in the immune system. Ann NY Acad Sci 1988 ; $524: 240-51$.

VanRollins $M$, Horrocks $L$, Sprecher $H$. Metabolism of 7,10,13,16-docosatetraenoic acid to dihomo-thromboxane, 14-hydroxy7,10,12-nonadecatrienoic acid and hydroxy fatty acids by human platelets. Biochim Biophys Acta 1985 ; 833 : 272-80.

Werz O, Bürkert E, Samuelsson B, Rådmark O, Steinhilber D. Activation of 5-lipoxygenase by cell stress is calcium independent in human polymorphonuclear leukocytes. Blood 2002 ; 99 : 1044-52.

Wu KK, Papp AC, Manner CE, Hall ER. Interaction between lymphocytes and platelets in the synthesis of prostacyclin. J Clin Invest 1987 ; 79 : 1601-6.

Yamamoto K, Ninomiya $\mathrm{Y}$, Iseki $\mathrm{M}$, et al. 4-Hydroxydocosahexaenoic acid, a potent peroxisome proliferator-activated receptor gamma agonist alleviates the symptoms of DSS-induced colitis. Biochem Biophys Res Commun 2008 ; 367 : 566-72. 